\title{
A Comparative Study of Automatic Extraction of Collocations from Corpora: Mutual Information vs. Cost Criteria
}

\author{
Kenji Kita, ${ }^{\dagger}$ Yasuhiko Kato, ${ }^{\dagger \dagger}$ Takashi Omoto ${ }^{\dagger}$ and Yoneo Yano ${ }^{\dagger}$
}

\begin{abstract}
While corpus-based studies are now becoming a new methodology in natural language processing, second language learning offers one interesting potential application. In this paper, we are primarily concerned with the acquisition of collocational knowledge from corpora for use in language learning. First we discuss the importance of collocational knowledge in second language learning, and then take up two measures, mutual information and cost criteria, for automatically identifying or extracting collocations from corpora. Comparative experiments are made between the two measures using both Japanese and English corpora. In our experiments, the cost criteria measure proved more effective in extracting interesting collocations such as fundamental idiomatic expressions and phrases.
\end{abstract}

KeyWords: Corpus, Collocations, Mutual Information, Cost Criteria, Second Language Learning

\section{Introduction}

Recent rapid advances in computer technology, especially the advent of large storage devices and parallel computers, and numerous data collection efforts have caused a shift in natural language applications from a knowledge-based to a corpus-based or data-intensive approach. The knowledge-based approach focused on abstraction of language, describing linguistic phenomena through minimal core knowledge such as parts-of-speech, syntactic and semantic rules. Linguistic phenomena, however, vary so vastly that they cannot be described through core knowledge. In addition, hand-coding knowledge takes a lot of time and hard work. The knowledge-based approach, therefore, has been found wanting in developing largescale practical NLP systems.

On the other hand, the corpus-based approach makes no claim about the compactness of the knowledge. Rather, the corpus-based approach derives more power from massive quantities of textual data than from hand-coded knowledge, being able to compensate for the weakness of the knowledge-based approach through authentic examples and various statistics of language use. With the availability of large corpora in recent years, many successful results have

$\dagger$ Tokushima University, Faculty of Engineering

†† The National Language Research Institute, Section for Dictionary Research 
been derived from corpus-based studies. These include part-of-speech tagging (Kupiec 1992), parsing (Magerman and Marcus 1990), example-based machine translation(Sumita and Iida 1992), statistical machine translation (Brown, Cocke, Pietra, Pietra, Jelinek, Lafferty, Mercer, and Roossin 1993; Brown, Pietra, Pietra, and Mercer 1993), language modeling (Jelinek 1990; Kita 1992) and many other related areas.

One interesting potential use of corpora is for second language learning. Kita et al. (Kita, Hayashi, and Yano 1993a) discussed various way of using corpora in language learning, which includes translation skill acquisition through bilingual corpora, oral/aural skill acquisition through speech corpora, multimedia language learning through structured corpora, and so forth. The greatest advantage of using corpora in language learning is that the corpora provide a body of evidence for the function and usage of words and expressions. At the same time, deriving lexical knowledge from large-scale corpora via automated procedures, as well as its use in language learning CAI systems, is one of the most important issues.

In this paper, we are primarily concerned with the acquisition of collocational knowledge from corpora. In Section 2, we describe why collocational knowledge is important in second language learning. In Section 3, we discuss the automatic extraction of collocations, taking up two measures, mutual information and cost criteria, for identifying or extracting collocations from corpora. In Section 4, we describe comparative experiments in extracting collocations and discuss the two measures.

\section{Importance of Collocational Knowledge in Language Learning}

There has been much theoretical and applied research on collocations, both from a linguistic and an engineering point of view. Consequently, the definition of collocation differs according to the researcher's interest and standpoint. This paper adopts the most comprehensive definition: a collocation is a cohesive word cluster, including idioms, frozen expressions and compound words.

The importance of collocations has been stressed in an extensive literature. From a language learning viewpoint, it can be summarized as follows:

- In language learning, learners must pay attention to how words are used rather than to individual words by themselves. Collocational knowledge indicates which words cooccur frequently with other words and how they combine within a sentence. Therefore, collocational knowledge is especially effective in sentence generation (Smadja and McK- 
eown 1990).

- Collocational knowledge is very difficult to acquire for second language learners. A typical example is the pair of words "strong" and "powerful" (Church, Gale, Hanks, and Hindle 1991; Smadja 1991). These two words have similar meanings, but their usage is quite different. For example, native English speakers prefer saying "strong tea" to "powerful tea", and prefer saying "powerful car" to "strong car". For non-natives, however, it is difficult to catch the subtle distinctions between these two words. These lexical preferences were sometimes ignored in the traditional knowledge-based approach; nevertheless they are the most important source for word choice and word ordering.

- It is pointed out that human translation process is based on analogical thinking (Nagao 1984). First, a human translator properly decomposes a sentence into certain fragmental phrases, then s/he translates each fragmental phrase by analogy with other examples, and finally composes fragmental translations into one sentence. Recently, following this idea, example-based machine translations (EBMT) have been widely explored. In some EBMT systems, several kinds of translation knowledge are utilized, such as the string-, pattern-, and grammar-level knowledge types. Collocations are particularly suitable for translation units in either the string- or pattern-level knowledge.

- From a cognitive point of view, it is said that human language acquisition is governed by the law of maximal efficiency (Wolff 1991). In other words, data compression, often called chunking, is performed to minimize storage demands in the brain. A chunk is considered to be a pattern which repeatedly appears in a variety of contexts. Collocations are good candidates for chunk units.

\section{Extracting Collocations from Corpora}

In the past, several approaches have been proposed to extract collocations from corpora. Church et al. (Church and Hanks 1990; Church et al. 1991) introduced the association ratio, which indicates how strongly two words are related, based on the information-theoretic concept of mutual information. Smadja et al. (Smadja and McKeown 1990; Smadja 1991) take into account word distance as well as word strength for a measure of word association. Also, Basili et al. (Basili, Pazienza, and Velardi 1992) proposed a syntax-based approach. Particularly, mutual information plays a central role in recent lexical statistical research. To take a few examples, Hindle and Rooth (Hindle and Rooth 1993) applied mutual information to disambiguate prepositional phrase attachments, and Brown et al. (Brown, Pietra, deSouza, Lai, and Mercer 1992) used it in determining word classes. 
In this section, after surveying how mutual information can be used to extract collocational information, we introduce another measure, called cost criteria (Kita, Ogura, Morimoto, and Yano 1993b), to automatically extract interesting collocations from corpora. Comparative experiments and discussions will be described in the next section.

\subsection{Mutual Information}

The mutual information between two words $x$ and $y$ is defined as follows (Church and Hanks 1990; Church et al. 1991):

$$
I(x, y)=\log \frac{P(x, y)}{P(x) P(y)}
$$

Here, $P(x)$ and $P(y)$ are word occurrence probabilities, and can be estimated from the number of occurrences of the words, $f(x)$ and $f(y)$, and the number of words in the corpus, $N$.

$$
P(x)=\frac{f(x)}{N} \text { and } P(y)=\frac{f(y)}{N}
$$

$P(x, y)$, the joint probability of $x$ and $y$, is estimated in a similar way.

$$
P(x, y)=\frac{f(x, y)}{N}
$$

where $f(x, y)$ is the number of occurrences of $x$ followed by $y$.

The mutual information $I(x, y)$ compares the probability of observing $x$ and $y$ together with the probabilities of observing $x$ and $y$ simply by chance. Thus, a large value indicates that the two words $x$ and $y$ have a strong relationship. By extracting word pairs with large mutual information values, we can obtain common collocations.

Because mutual information values are defined for two words, this simple method can only extract collocations of length two. However, a generalization is suggested in (Jelinek 1990) as follows:

(1) Start out from the basic vocabulary $V_{0}$. Set $n=0$.

(2) Augment the vocabulary $V_{n}$ by all word sequences " $x y$ " for which $I(x, y)>T h r$, where $T h r$ is a predetermined threshold.

(3) From Step 2, a new vocabulary $V_{n+1}$ is established.

(4) Adjust the vocabulary size $N$ to reflect the new vocabulary $V_{n+1}$.

(5) Resume from Step 1 with $V_{n+1}$ as its basis.

With this iterative procedure, the final vocabulary includes collocations of arbitrary length. 


\subsection{Cost Criteria}

The cost criteria measure is based on the assumptions that (1) collocations are recurrent word sequences, and (2) the recurrent property is captured by the absolute frequency of a word sequence. However, a simple absolute frequency approach does not work, because the frequency of a sub-sequence is always higher than that of the original word sequence. Therefore, as a measure for identifying or extracting collocations, absolute frequency is not appropriate. Instead, we consider a processing cost for a word sequence, and introduce cost criteria which can quantitatively estimate the extent to which processing is reduced by considering a word sequence as one unit.

Before the presentation of a formal definition, we introduce the following notation:

$$
\begin{aligned}
& \begin{array}{lll}
\alpha & \cdots & \text { a word sequence. }
\end{array} \\
& |\alpha| \quad \cdots \quad \text { the length of } \alpha \text {. } \\
& \text { (the number of words in } \alpha \text { ) } \\
& f(\alpha) \quad \cdots \quad \text { number of occurrences of } \alpha \text { in a corpus. }
\end{aligned}
$$

We define a reduced cost $K(\alpha)$ for a sequence $\alpha$ as:

$$
K(\alpha)=(|\alpha|-1) \times f(\alpha)
$$

$K(\alpha)$ is interpreted as follows. Assume here that, in the corpus, there exists a word sequence $\alpha$, which is composed of $|\alpha|$ words and occurs $f(\alpha)$ times. Also assume that the cost of processing one word is 1 . Similarly, when processing $\alpha$ as a single unit, its processing cost is 1 . Here, we assume that: if a word sequence is processed one word at a time, its processing cost is proportional to its length. This assumption is made because of the following reason. We are now concerned with the problem of identifying the sequence $\alpha$ as a collocation. But since this problem is reduced to a string pattern matching problem, it is natural to assume that the cost is proportional to the length of the sequence $\alpha$. That is, the processing cost for $\alpha$ is $|\alpha|$. By considering $\alpha$ as one unit, the processing cost is reduced to $|\alpha|-1$. Since $\alpha$ appears $f(\alpha)$ times, we can conclude that the total reduced cost becomes $(|\alpha|-1) \times f(\alpha)$, which is the definition of $K(\alpha)$.

In reality, however, the problem is not so simple, because word sequences are not mutually disjoint. Consider the case where a word sequence $\alpha$ is a sub-sequence of $\beta$ (for example, $\alpha=$ "in spite", $\beta=$ "in spite of"). Then, we have:

$$
f(\alpha) \geq f(\beta)
$$

Further, the word sequence $\alpha, f(\beta)$ times out of $f(\alpha)$ times, will be identified as $\beta$. Thus, the 
actual reduced cost for $\alpha$ is defined as:

$$
K(\alpha)=(|\alpha|-1) \times(f(\alpha)-f(\beta))
$$

Finally, we can extract collocations from a corpus by the following steps:

(1) Calculate $K(\alpha)$ for each word sequence $\alpha$ in a corpus.

(2) Rank a word sequence $\alpha$ by using the value $K(\alpha)$.

(3) Extract higher rank word sequences as collocation candidates.

(4) Re-calculate $K(\alpha)$ for each $\alpha$ in the collocation candidates. To be more precise, by checking the sequence/sub-sequence relation between every two word sequences in the collocation cancidates, modify the $K(\alpha)$ values according to Equation 9 .

\section{Experiments and Discussions}

\subsection{The ADD Corpus}

In our experiments, the ADD (ATR Dialogue Database) Corpus (Ehara, Ogura, and Morimoto 1990) created by ATR Interpreting Telephony Research Laboratories in Japan was used. The ADD Corpus is a large structured database of dialogues collected from simulated telephone or keyboard conversations which are spontaneously spoken or typed in Japanese or English. This corpus consists of parallel texts of Japanese and English, aligned by utterance. Also, sentences in ADD are morphologically analyzed and annotated with various kinds of syntactic, semantic, and phonological information.

Currently, the ADD Corpus contains textual data from two tasks (text categories); one consists of simulated dialogues between a secretary and participants at international conferences (Conference Task), and the other of simulated dialogues between travel agents and customers (Travel Task).

In our experiments, we used the keyboard dialogues from the Travel Task, which include approximately 120,000 Japanese words and 100,000 English words. The telephone dialogue include linguistic phenomena, such as filled pauses ("ah", "uh", etc.), restarts (repeating a word or phrase) and interjections, so we did not use them for our experiments. The aim in our research is to extract linguistically neat expressions, thus we excluded telephone dialogues from the experiments.

\subsection{Results and Discussions}

Table 1 shows some interesting Japanese collocations extracted using respectively mutual information and cost criteria. Table 2 shows some English ones. In these tables, collocations 
are listed in descending order with respect to their values (i.e. mutual information or cost reduction values).

In the experiments, we examined word sequences up to a 10 word length. When using mutual information, the threshold was set to 0.0. This means that we calculated mutual information values for all the word sequences which appeared in the corpus. When using cost criteria, we generated approximately 10,000 collocation candidates. The collocations listed in the tables were selected manually from the higher ranked ones.

Before discussing the results, we first overview the characteristics of Japanese phrases. In general, the order of major constituents in a Japanese sentence is rather free. However, predicate phrase positioning is dominated by the so-called predicate-phrase ending constraint: a predicate phrase appears at the end of its clause. Furthermore, a predicate phrase often has a complex form, consisting of a main predicate such as a verbal noun, verb or adverb, combinations of auxiliary predicates, and a sentence-final particle. These auxiliary predicates and sentence-final particles add various complementary meanings to a sentence, such as honorific, causative, and prohibitive meanings, etc.

As can be seen from the experimental results (Table 1), the method based on mutual information tends to extract task-dependent compound noun phrases, while cost criteria tends to extract complex predicate phrase patterns. Almost all the collocations extracted are in this category. For example, the collocations "desho u ka" and "desu ka", which had a high cost reduction, are used very often to make interrogative sentences in Japanese. The collocation "tai no desu ga" is usually used to express a speaker's request, whose meaning is "(I) would like to".

The comments above are also true of the English data. Mutual information tends to extract compound noun phrases, while cost criteria tends to extract frozen phrase patterns such as "thank you very much" and "I would like to".

Why does mutual information fail to extract these patterns? Here, let us take "I will" as an illustrative example, which has been picked out by cost criteria ("I will" is omitted from Table 2) but not by mutual information. In our corpus, "I" occurs 2,907 times, "will" occurs 920 times, and "I will" occurs 264 times. Therefore, we have

$$
\begin{aligned}
I(\mathrm{I}, \text { will }) & =\log \frac{\frac{264}{100,000}}{\frac{2907}{100,000} \frac{920}{100,000}} \\
& =3.3
\end{aligned}
$$

This value is not so large, so the two words "I" and "will" cannot be considered to have a significant relationship. 
According to the same reasoning, patterns such as "I would like to" and "thank you very much" are excluded as collocation candidates. However, in the ADD Corpus, more than fifty per cent of the sentences that involve the word "would" are subsumed under the pattern "(I) would like to $\sim$ ". Therefore, this pattern should be included in the collocation list.

Another drawback using mutual information is the sparseness of data. A corpus cannot provide sufficient data about every word-word relationship. Some word pairs may have high mutual information values in spite of their low frequency in the corpus. For example, the first ranked collocation was "yacht harbor", which occurs only twice in the ADD Corpus. On the contrary, since the cost criteria measure is based on absolute frequency, such phenomena never happens.

Now, let us consider why mutual information tends to extract task-dependent compound nouns. As noted above, mutual information values become unstable for low-frequency words. Consequently, even if the incidence of a word pair occuring is low, it is quite possible that the mutual information value becomes greater. These low-frequency words, however, often depend on the topic of the text. This is one reason why mutual information tends to extract task-dependent compound nouns.

\section{Conclusion}

With the growing availability of large textual resources, corpus-based studies are gaining more and more attention among computational linguists and computer scientists. In particular, automatic acquisition of lexical knowledge from corpora is one of the most important and interesting issues. In this paper, we have taken up the problem of how to acquire collocational knowledge and discussed its importance for language learning. We have also described comparative experiments using mutual information and cost criteria. Our experiments demonstrated that mutual information tends to extract task-dependent compound noun phrases, while cost criteria tends to extract predicate phrase patterns.

Unfortunately, the current implementation can only extract collocations of uninterrupted word sequences. Our next plan is to refine the method to extract collocations of interrupted sequences, and to utilize lexical information such as parts-of-speech in order to prevent an improper word sequence from being recognized as a collocation. For example, when applying cost criteria to English corpora, unwelcome word pairs, such as "on the", "for the" or "of the", are extracted because such pairs occur frequently in the language. However, these word pairs can be excluded by using parts-of-speech information. Also, we hope to incorporate extracted 
collocations into a language learning CAI system.

Acknowledgement The idea of cost criteria was developed while the first author was staying at ATR Interpreting Telephony Research Laboratories. The authors are deeply grateful to co-researchers in ATR, Kentaro Ogura (currently with NTT Network Information Systems Laboratories) and Tsuyoshi Morimoto, for their fruitful discussions and comments. The authors are also grateful to the members of our laboratory in Tokushima University for their help and encouragement. Special thanks to Gerardo Ayala, Ingrid Kirschning and John Phillips for reading the manuscript.

\section{Reference}

Basili, R., Pazienza, M. T., and Velardi, P. (1992). "A shallow syntactic analyzer to extract word associations from corpora." Literary and Linguistic Computing, 7(2), 113-123.

Brown, P. F., Cocke, J., Pietra, S. A. D., Pietra, V. J. D., Jelinek, F., Lafferty, J. D., Mercer, R. L., and Roossin, P. S. (1990). "A statistical approach to machine translation." Computational Linguistics, 16(2), 79-85.

Brown, P. F., Pietra, S. A. D., Pietra, V. J. D., and Mercer, R. L. (1993). "The mathematics of statistical machine translation: Parameter estimation." Computational Linguistics, 19(2), 263-311.

Brown, P. F., Pietra, V. J. D., deSouza, P. V., Lai, J. C., and Mercer, R. L. (1992). "Classbased n-gram models of natural language." Computational Linguistics, 18(4), 467-479.

Church, K. W., Gale, W., Hanks, P., and Hindle, D. (1991). "Using statistics in lexical analysis." In Zernik, U. (Ed.), Lexical Acquisition: Exploiting On-Line Resources to Build a Lexicon, pp. 115-164. Lawrence Erlbaum Associates.

Church, K. W., and Hanks, P. (1990). "Word association norms, mutual information, and lexicography." Computational Linguistics, 16(1), 22-29.

Ehara, T., Ogura, K., and Morimoto, T. (1990). "ATR dialogue database." In Proc. of the 1990 International Conference on Spoken Language Processing, pp. 1093-1096.

Hindle, D., and Rooth, M. (1993). "Structural ambiguity and lexical relations." Computational Linguistics, 19(1), 103-120.

Jelinek, F. (1990). "Self-organized language modeling for speech recognition." In Waibel, A., and Lee, K. F. (Eds.), Readings in Speech Recognition, pp. 450-506. Morgan Kaufmann Publishers.

Kita, K. (1992). A Study on Language Modeling for Speech Recognition. Ph.D. thesis, Waseda 
University.

Kita, K., Hayashi, T., and Yano, Y. (1993a). "Corpus-based language learning: Towards practical language learning systems." In Proc. of the 1993 International Conference on Computers in Education, pp. 355-357.

Kita, K., Ogura, K., Morimoto, T., and Yano, Y. (1993b). "Automatically extracting frozen patterns from corpora using cost criteria." Transactions of Information Processing Society of Japan, 34(9), 1937-1943. (in Japanese).

Kupiec, J. (1992). "Robust part-of-speech tagging using a hidden Markov model." Computer Speech and Language, 6, 225-242.

Magerman, D. M., and Marcus, M. P. (1990). "Parsing a natural language using mutual information statistics." In Proc. of the Eight National Conference on Artificial Intelligence, pp. 984-989.

Nagao, M. (1984). "A framework of a mechanical translation between Japanese and English by analogy principle." In Elithorn, A., and Banerji, R. (Eds.), Artificial and Human Intelligence, pp. 173-180. Elsevier Science Publishers.

Smadja, F. A. (1991). "Macrocoding the lexicon with co-occurrence knowledge." In Zernik, U. (Ed.), Lexical Acquisition: Exploiting On-Line Resources to Build a Lexicon, pp. 165-189. Lawrence Erlbaum Associates.

Smadja, F. A., and McKeown, K. R. (1990). "Automatically extracting and representing collocations for language generation." In Proc. of the 28th Annual Meeting of the Association for Computational Linguistics, pp. 252-259.

Sumita, E., and Iida, H. (1992). "Example-based NLP techniques: A case study of machine translation." In Proc. of the AAAI Workshop on Statistically-Based NLP Techniques, pp. 90-97.

Wolff, J. G. (1991). Towards a Theory of Cognition and Computing. Ellis Horwood. 
Table 1 Some examples of extracted collocations. (Japanese)

\begin{tabular}{l|l}
\hline \multicolumn{1}{c|}{ Mutual Information } & \multicolumn{1}{c}{ Cost Criteria } \\
\hline ichi ryuu no orchestra ni yoru ensou & desho u ka \\
Jouzankei-onsen to set ni nat ta golf-pack & desu ka \\
Kunitachi-shi Ishida & desho u \\
chijou e & mashi ta \\
night-tour ya dinner-show & sou desu \\
kaihatsu ga sakan & sou desu ka \\
buchou ya kachou & to iu koto \\
$\begin{array}{l}\text { mai tsuzuri } \\
\text { hizuke henkou sen wo koe }\end{array}$ & sou desu ne \\
moushikomi kin toshite o azukari & masu ka \\
Shinjuku-ku Naitou-chou 1 banchi & desu ne \\
kokunai sen no daiya & o negai shi masu \\
hakkou kaisha ni teishutsu & itashi masu \\
Kenya Tanzania Safari & o negai itashi masu \\
yuuran sen no senchou & to omoi masu \\
kaisui yoku & te ori masu \\
yuukyuu kyuuka & tai no desu ga \\
Matsushima-wan meguri & wakari mashi ta \\
resort kaihatsu & kashikomari mashi ta \\
umi to yama & ni nari masu \\
yuujin no hahaoya & to iu no ha \\
Hachiman-daira Towada Hiraizumi & shi tai no desu ga \\
danjo betsu no uchiwake & to iu koto de \\
hakubutsu kan & na n desu ga \\
dou nenpai & shi tai no desu \\
senmon yougo & shouchi itashi mashi ta \\
yuukou kigen & to iu koto desu \\
genkin kakitome & sou na n desu \\
Shanghai Sian & arigatou gozaimashi ta \\
Setagaya-ku Kyoudou & sa se te itadaki masu \\
seinen gappi & o mata se itashi mashi ta \\
moyori no eki & sou na n desu ka \\
choushoku to chuushoku & shitsurei itashi masu \\
Fuji-ginkou honten & yoroshii desho u ka \\
gouka kyakusen & ka mo shire mase n \\
\hline & irasshai masu ka \\
\hline
\end{tabular}


Table 2 Some examples of extracted collocations. (English)

\begin{tabular}{|c|c|}
\hline Mutual Information & Cost Criteria \\
\hline yacht harbor & is that so \\
\hline Echigo Yuzawa & thank you very much \\
\hline Fifth Avenue & I would like to \\
\hline General Affairs & I see \\
\hline Mitsuboshi trading & my name is \\
\hline slide projector & sorry to have kept you waiting \\
\hline strong background & is that right \\
\hline cross the International Date & in that case \\
\hline the F1 Grand Prix & I understand \\
\hline Shiretoko Sightseeing Boat Inc. & thank you for \\
\hline it's my pleasure & do you have any \\
\hline at the Hotel New Tanda & good bye \\
\hline give a speech & would you like to \\
\hline head Mr. Kuwata & I am very sorry \\
\hline wine production & a little \\
\hline Wall Street & be able to \\
\hline jazz dance & I got it \\
\hline my mother in law & I'll be waiting for your call \\
\hline to the historic sites & may I have your name and address \\
\hline I am not that familiar & how much \\
\hline Keirin and Peking & all right \\
\hline cause the inconvenience & as soon as possible \\
\hline holding a paper & then would you give me your \\
\hline baths and toilets & the other day \\
\hline Las Vegas & make the reservations \\
\hline Queen Elizabeth & a lot of \\
\hline Main Branch & I will call you \\
\hline Sales Department & that's right \\
\hline self introduction & how about \\
\hline zip code & at that time \\
\hline international cards & the application fee \\
\hline to the Grand Canyon & is that okay \\
\hline The Hyatt Regency & I appreciate your \\
\hline flight number JS & of course \\
\hline Canadian Rockies and Vancouver & so please hold the line \\
\hline
\end{tabular}


Kenji Kita: Kenji Kita received the B.S. degree in mathematics and the Ph.D degree in electrical engineering, both from Waseda University, Tokyo, Japan, in 1981 and 1992, respectively. From 1983 to 1987, he worked for the Oki Electric Industry Co. Ltd., Tokyo, Japan. From 1987 to 1992, he was a researcher at ATR Interpreting Telephony Research Laboratories, Kyoto, Japan. Since 1992, he has been with the Faculty of Engineering, Tokushima University, Tokushima, Japan, where he is currently an Associate Professor. $\mathrm{He}$ is a member of the Acoustical Society of Japan, the Information Processing Society of Japan, the Institute of Electronics, Information and Communication Engineers, and the Association for Computational Linguistics. His current research interests include speech recognition, natural language processing, and corpus linguistics.

Yasuhiko Kato: Yasuhiko Kato received the B.A. degree from Seijo University and the M.A. degree from Saitama Univeristy, both in linguistics. From 1984 to 1991, he worked for the Oki Electric Industry Co. Ltd., Tokyo, Japan. Among these years, also worked as a member of CICC and EDR. Since 1991, he has been a researcher at the National Language Research Institute, Tokyo, Japan. He is a member of the following societies: the Linguistic Society of Japan, the Society for the Study of Japanese Language, the Folklore Society of Japan, the Information Processing Society of Japan and the Association for Computational Linguistics. His current research interests include semantics and lexicology for dictionary description, corpus linguistics and natural language processing.

Takashi Omoto: Takashi Omoto received the B.E. degree from Tokushima University, Tokushima, Japan, in 1994. He is currently a master course student in Tokushima University.

Yoneo Yano: Yoneo Yano received the B.E., M.E., and Ph.D degrees in communication engineering from Osaka University, Osaka, Japan, in 1969, 1971, and 1974, respectively. Since 1974 he has been with Faculty of Engineering, Tokushima University, Tokushima, Japan. He is currently a Professor in Information Science and Intelligent Systems. 